Boise State University

ScholarWorks

$1-24-2014$

Reproducible Nanostructure Fabrication Using Atomic Force Microscopy Indentation with Minimal Tip Damage

SeungHee Jeon

Seoul National University

BongWoo Ryu

Seoul National University

Wonho Jhe

Seoul National University

Zheong G. Khim

Seoul National University

Byung I. Kim

Boise State University 


\title{
LETTERS
}

\section{Reproducible nanostructure fabrication using atomic force microscopy indentation with minimal tip damage}

\author{
SeungHee Jeon, BongWoo Ryu, Wonho Jhe, and Zheong G. Khim \\ Department of Physics and Astronomy, Seoul National University, Seoul 151-742, Republic of Korea \\ Byung I. Kim ${ }^{\text {a) }}$ \\ Department of Physics, Boise State University, Boise, Idaho 83725
}

(Received 6 November 2013; accepted 7 January 2014; published 21 January 2014)

\begin{abstract}
A uniform pattern of quantum dots and nanowires were reproducibly fabricated by creating holes in a two-layer structure using atomic force microscopy (AFM) indentation, dry-etching of polymer resists, and metal deposition through the indentation holes. The two-layer structure was created by depositing a thin gold layer onto a polymethyl methacrylate (PMMA) layer on a silicon substrate. The indentation depth was set so that the AFM tip penetrated the thin gold layer without the tip contacting the silicon substrate. This two-layer indentation was used to create a pattern of holes in the thin gold layer. Then, the PMMA was exposed to an isotropic $\mathrm{O}_{2}$ plasma etchant through the holes in the indentation pattern to form an undercut between the substrate and the gold layer. Quantum dots were subsequently created through the deposition of gold on the exposed silicon substrate through the indentation holes. Gold nanowires were also fabricated by creating indentation holes consecutively and optimizing the distance between the holes using the same two-layer indentation method. The topographic and electrical measurements of the fabricated gold nanowires suggest that our method is capable of making uniform and reproducible nanowires. The scanning electron microscopy images of the tips confirmed that the consecutive-hole-indentation method is less invasive than the conventional ploughing method, where constant tip contact occurs with the substrate during the formation of nanowires. (C) 2014 American Vacuum Society. [http://dx.doi.org/10.1116/1.4862538]
\end{abstract}

\section{INTRODUCTION}

As the size of electronic devices gets smaller, many methods of fabrication of nanoelectronic devices have been developed using nanolithography techniques such as electron-beam lithography (EBL), ${ }^{1} \mathrm{x}$-ray lithography, ${ }^{2}$ focused ion beam lithography (FIBL), ${ }^{3}$ and atomic force microscopy (AFM) nanolithography. ${ }^{4}$ Among these techniques, nanolithography using AFM is a more versatile method when compared to the EBL or FIBL. It performs inexpensively in a comparatively simple process, and it is able to fabricate nanostructures without risk of sample damage. However, it has disadvantages, such as poor reproducibility due to tip damage and low throughput due to the difficulty of application over a large area. Yet, this method is able to reproduce nanostructures at specified locations, which makes it useful when applied to nanosystems because it can easily make various patterns.

In the AFM nanolithography method, oxidation, ${ }^{5-7}$ thermomechanical writing, ${ }^{8}$ and indentation (direct mechanical lithography) are currently developed. Among them, AFM indentation nanolithography is a method where the AFM tip applies mechanical forces to deform the sample surface at a specified position in creating nanoscale patterns such as indentation holes and line grooves. ${ }^{9-13}$ The size and form of the AFM tip apex and the magnitude of the applied force are critical to determining

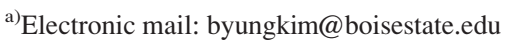

the size and the position of the patterns. While nanostructures have been easily built by this AFM indentation nanolithography utilizing the sharp AFM tip, tip damage from the indentation process limits the number of patterns that one tip can make reproducibly. To overcome this limitation, special tips, such as diamond-coated tips, are occasionally used. ${ }^{14,15}$

If the indentation is made on a single polymer layer, the tip applies vertical force directly to the substrate ${ }^{16-18}$ Although this single-layer indentation method could make nanostructures, the AFM tip could be easily damaged because the tip applies the force to the hard substrate directly while making indentations. The ploughing method, which also uses an AFM, is another way to simply fabricate nanowires, but the AFM tip can be easily damaged because of the lateral force applied to it. While Wiesauer et al. showed that by reducing the distance between the holes, line grooves could be created, ${ }^{9}$ the fabrication of line grooves using the ploughing method also has limitations because of the tip damage from the single-layer structure on a hard substrate mentioned above. Once the tip apex is damaged, the width and depth of the indentation pattern will vary even if the same loading force is applied. The damaged tip apex makes it harder to create reproducible and uniform nanostructures using the conventional ploughing method.

In our experiment, we used a two-layer structure to improve the reproducibility of a uniform nanostructure, which was made through the deposition of a soft thin metal layer on the polymer surface. ${ }^{19}$ In the two-layer structure, the depth of 
the indentation pattern was set to be deeper than the thickness of the soft thin metal layer, but not deep enough for the tip to contact the substrate directly, thereby preventing the tip damage..$^{19}$ As an inherent drawback of this method, it requires extra processes such as metal deposition and plasma etching. While the extra processes add complexity and extra cost compared to a straightforward ploughing process, the method improves the uniformity and reproducibility of nanostructure. It also drops the cost of tip damage. Using the new method, we fabricated quantum dots and gold nanowires by creating indentation holes consecutively under the optimal adjustment of distances between neighboring holes.

\section{EXPERIMENT}

In this experiment and all subsequent experiments, the lateral speed of the AFM tip and the $\mathrm{z}$ direction speed of the AFM scanner were fixed to $5 \mu \mathrm{m} / \mathrm{s}$ during the indentation. Polymethyl methacrylate (PMMA) (950k A2, Microchem, Japan) was spin-coated at $5000 \mathrm{rpm}$ for $40 \mathrm{~s}$ on thermally grown $100 \mathrm{~nm}$ thickness of $\mathrm{SiO}_{2-\mathrm{x}}$ on a $10 \times 10 \mathrm{~mm}^{2}$ size of silicon substrate, then baked in oven at $170^{\circ} \mathrm{C}$ for $30 \mathrm{~min}$. Gold was then deposited as a soft metal mask onto the PMMA layer with the thickness of $\sim 15 \mathrm{~nm}$ [Fig. 1(a)]. The thickness of the PMMA film and the root mean square values of the final gold-PMMA film were $80 \mathrm{~nm}$ and $0.45 \mathrm{~nm}$, respectively. The loading force applied on the Au-PMMA surface by the AFM tip was $2-3.5 \mu \mathrm{N}$. All experiments were carried out at room temperature with a relative humidity of $40-60 \%$.

The indentation was carried out using a commercial AFM (Albatross II, NanoFocus, Korea), and a commercial noncontact silicon cantilever with a tetrahedral shape (NSG30, NT-MDT, Russia). The cantilever has a force constant of $40 \mathrm{~N} / \mathrm{m}$ and a resonant frequency of 310-320 kHz. Each experiment was conducted with a new tip. The cantilever was first moved to a specified position on the two-layer sample surface in noncontact mode. After the feedback loop was disabled, the indentation

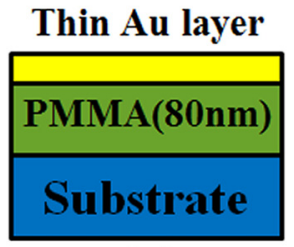

(a)

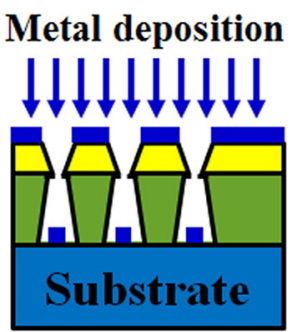

(c)

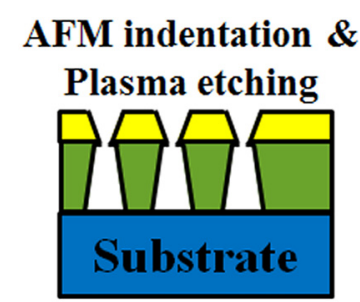

(b)

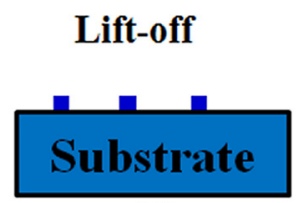

(d)
FIG. 1. (Color online) Schematic diagram of the experimental procedure. process was performed. While the sample was raised to a preset height, the tip of the cantilever contacted and indented the sample surface. As soon as the sample reached the preset height, it retracted to the starting position using the piezotube. These steps were repeated by moving the tip to other positions until a $40 \times 40$ quantum dot structure was created. Then, an isotropic $\mathrm{O}_{2}$ plasma etching was applied to the indented surface by PR Asher (V15-G, Plasma Finish, Germany) at base pressure $3.0 \times 10^{-3}$ Torr, operation pressure $7.0 \times 10^{-3}$ Torr, and plasma power $120 \mathrm{~W}$ for $70-80 \mathrm{~s}$ [Fig. 1(b)]. The PMMA exposed to the plasma etchants was removed, while the PMMA beneath the indentation pattern formed an undercut due to the isotropic $\mathrm{O}_{2}$ plasma etching. The undercut made it easy to remove the PMMA residue where the metal had been deposited. This guaranteed a clean contact without polymer residue between the nanostructure and the substrate and made the lift-off easier. Although it was possible to deposit the metal in less than $70 \mathrm{~s}$, a total minimum etching time of 70-80 s was needed to ensure the undercut formation. By this process, a metal mask having indentation holes and line grooves with the depth and width of a few tens of nanometers was fabricated. Gold was then used as the evaporation metal and deposited with an e-gun evaporator (VI-43N, Anelva, Japan) [Fig. 1(c)] at a thickness of $15-20 \mathrm{~nm}$. The base pressure was $3.0 \times 10^{-7}$ Torr. The thickness of the gold was controlled using a thin film thickness monitor (XTC, Infilcon, Switzerland). Lastly, the lift-off procedure was conducted in acetone with ultrasonic cleaner for 5 min [Fig. 1(d)].

We also fabricated two nanowires, $15 \mu \mathrm{m}$ in length, on a silicon surface using the same process described above. For comparison, two line grooves of the same length and depth were made using the ploughing method and the consecutivehole-indentation method by adjusting the distance between the holes. Then, a scanning electron microscopy (SEM) investigation was carried out on the used AFM tips.

The quantum dots and the nanowires were measured by noncontact mode AFM (SPA 400, Seiko, Japan) with a new cantilever of the same kind used for the indentation. The nanowire was also fabricated on the substrate with electrodes, which were made by standard photolithography methods, and then current-voltage characteristics were measured.

\section{RESULTS AND DISCUSSION}

Use Fig. 2(a) is the AFM image of the $40 \times 40$ indented hole pattern and a close-up image of the marked area on the thin gold layer-PMMA structures. The distances between the indented holes range from $100 \mathrm{~nm}$ to $130 \mathrm{~nm}$. Figure 2(b) shows the two line grooves $15 \mu \mathrm{m}$ in length and a close-up image of the marked area. This line pattern was made using the consecutive-hole-indentation method with a distance of $20 \mathrm{~nm}$ between the holes. The close-up image in Fig. 2(b) shows that the two line grooves were uniformly fabricated in this hole distance. The depth of the indented hole and the two line grooves is $\sim 20 \mathrm{~nm}$, which is deep enough to penetrate the thin gold film.

Figure 3(a) is the AFM image of the $40 \times 40$ gold quantum dot array result. The average full width at half maximum 


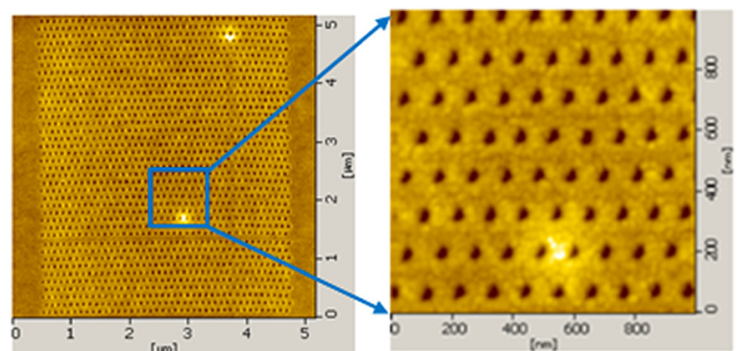

(a)

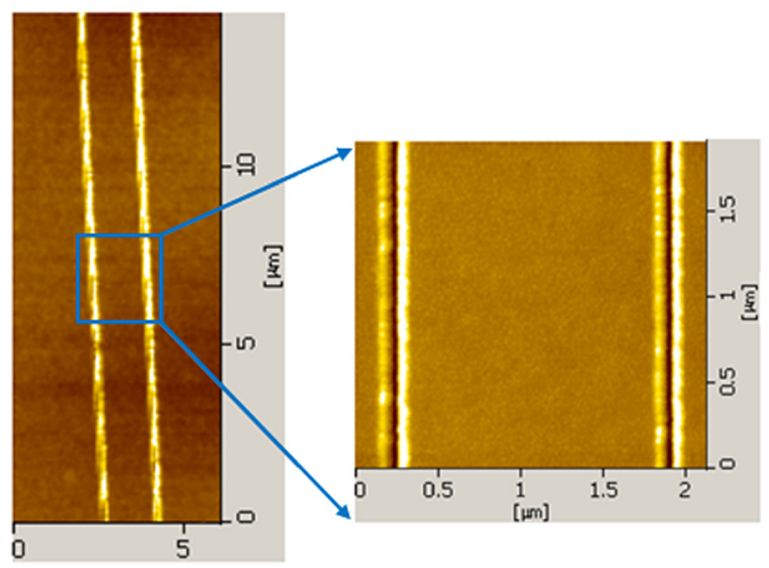

(b)

FIG. 2. (Color online) AFM images of (a) $40 \times 40$ indented hole pattern array and a close-up image of the marked area, and (b) $15 \mu \mathrm{m}$ length of linegroove pattern and a close-up image of the marked area.

(FWHM) value of the gold quantum dot shown in the inset of Fig. 3(b) was $42.4 \mathrm{~nm} \pm 1.3 \mathrm{~nm}$. The round shape of the gold quantum dot in Fig. 3(b) indicates that the nanostructure is not directly related to the shape of the tetrahedral tip. This result suggests that the quantum dot was developed through diffusion process, which determines the shape and resolution of the quantum dot. The dot size in Fig. 3(b) indicates that the quantum dots would connect if their distances were less than $50 \mathrm{~nm}$. To test this idea, two nanowires were fabricated when the indentation hole distance was set at $50 \mathrm{~nm}$. Contrary to our expectation, the two nanowires (Fig. 4) show that the dots are not connected completely. This result suggests that the gold film had not been completely removed between the two successive holes. The inset of Fig. 4 also shows that each dot was fabricated at intervals of $\sim 50 \mathrm{~nm}$, which is the indentation distance between the dent holes. This result indicates that the distance between the dent holes should be less than $\sim 40 \mathrm{~nm}$ for the fabrication of a uniform nanowire.

When the hole distance was changed to $20 \mathrm{~nm}$, we obtained two continuous line grooves [Fig. 2(b)]. When we repeated this experiment with hole distances less than $20 \mathrm{~nm}$, continuous line grooves were also obtained. The AFM image [Fig. 5(a)] shows a very uniform nanowire that was made using this method. The average FWHM value of the nanowire in Fig. 5(b) was $63.1 \mathrm{~nm} \pm 1.9 \mathrm{~nm}$ over 20 consecutive sectional profiles along the line grooves at intervals of $200 \mathrm{~nm}$ [Fig. 5(c)]. As a result, we found that the control of the

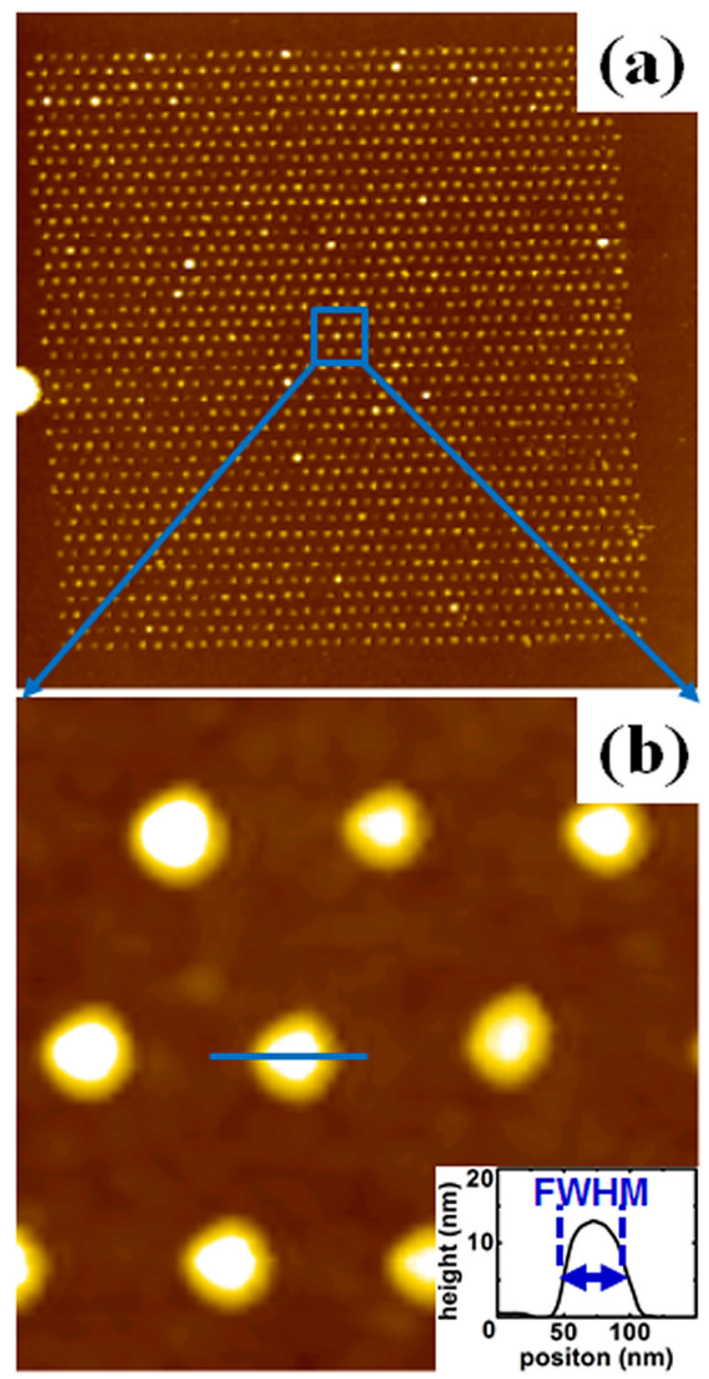

FIG. 3. (Color online) (a) AFM image of $40 \times 40$ gold quantum dots (scan area $6 \mu \mathrm{m} \times 6 \mu \mathrm{m}$ ), and (b) close-up image of the marked area in (a) (scan area $0.4 \mu \mathrm{m} \times 0.4 \mu \mathrm{m}$ ) [inset: cross section of the marked line in (b)].

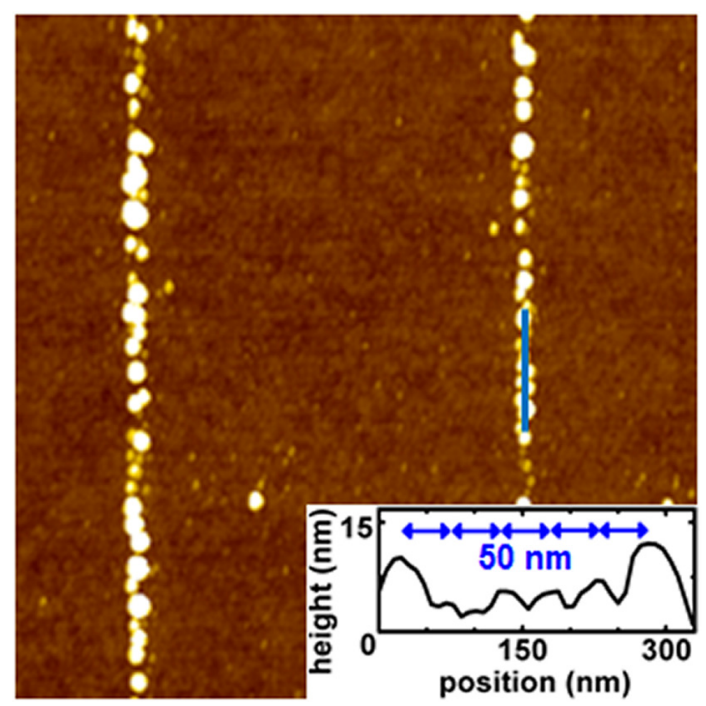

FIG. 4. (Color online) AFM image of gold nano dotted-line structure made by indentation with $50 \mathrm{~nm}$ distance between the holes (scan area $2 \mu \mathrm{m} \times 2 \mu \mathrm{m})$ (inset: cross section of the marked line). 


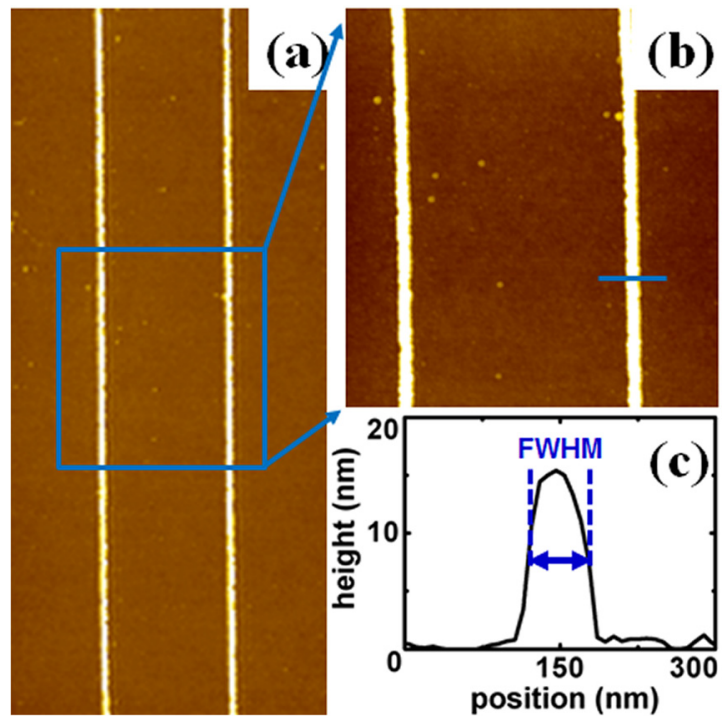

FIG. 5. (Color online) (a) AFM image of gold nanowire (scan area $3 \mu \mathrm{m} \times 6.5 \mu \mathrm{m}$ ), (b) close-up image of the marked area in (a) (scan area $2 \mu \mathrm{m} \times 2 \mu \mathrm{m}$ ), and (c) cross section of the marked line in (b).

distance between the holes is important to fabricating a uniform nanowire because adjusting the hole-hole distance minimized the tip damage by reducing unnecessary indentation.

A gold nanowire was fabricated under the same condition as the previous experiment in Fig. 5 and was connected to the electrodes on the substrate to study its electrical characteristics (Fig. 6). The electrode was fabricated by standard photolithography. The distance between the electrodes was about $10 \mu \mathrm{m}$ or $5 \mu \mathrm{m}$, and the height of the electrode was $40 \mathrm{~nm}$ [oblique line area in Fig. 6(a)]. Figure 6(b) shows four different measurements of the current versus voltage characteristics of the nanowires measured by the two-terminal method. We obtained the resistivities of the gold nanowires using this result by making three assumptions as follows: The first is that the contact resistances $\left(R_{C}\right)$ between the electrodes and the nanowire are the same at both ends; the second is that the size of the contact resistance is only inversely proportional to the area between the nanowire and electrode; the last assumption is that the circuit resistance is negligible except for the nanowire resistance $\left(R_{\mathrm{NW}}\right)$ and the contact resistance between the nanowire and electrode. According to the first and third assumptions, the total resistance of the system $(R)$ is written as follows:

$$
R=2 R_{C}+R_{\mathrm{NW}}
$$

Since the nanowire resistance is proportional to the ratio between the length and cross section area of the nanowire, it is written as follows:

$$
R_{\mathrm{NW}}=\rho \frac{l}{w \times h},
$$

where $l, w$, and $h$ are the length, width, and height of the nanowire, respectively. The proportional constant $\rho$ corresponds to the electrical resistivity. According to the second assumption, the contact resistance is written as follows:

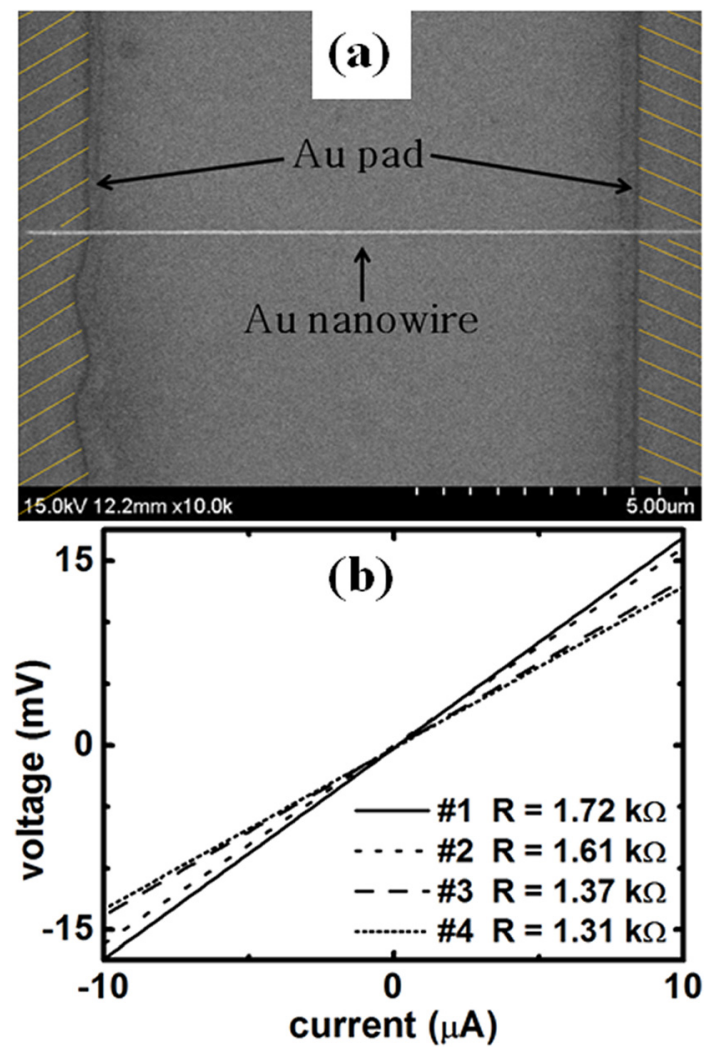

FIG. 6. (Color online) (a) FE-SEM picture of gold nanowire made on prefabricated electrode (oblique line area), and (b) voltage graph as a function of the electric current of gold nanowires. The length, width, and height of the nanowires are as follows. \#1: $10.4 \mu \mathrm{m}, 63 \mathrm{~nm}$, and $21 \mathrm{~nm}, \# 2: 10.2 \mu \mathrm{m}$, $62 \mathrm{~nm}$, and $21 \mathrm{~nm}, \# 3: 5.2 \mu \mathrm{m}, 49 \mathrm{~nm}$, and $20 \mathrm{~nm}$, and \#4: $5.2 \mu \mathrm{m}, 48 \mathrm{~nm}$, and $20 \mathrm{~nm}$, respectively.

$$
R_{C}=\frac{C}{w \times h},
$$

where $C$ is a constant. The combination of Eqs. (2) and (3) with Eq. (1) leads to the following equation:

$$
R=2 \frac{C}{w \times h}+\rho \frac{l}{w \times h} .
$$

As Eq. (4) has two unknowns, $C$ and $\rho$, two equations are required to determine them. The two equations were set up by choosing two nanowires, one from two $10 \mu \mathrm{m}$ nanowires and the other from two $5 \mu \mathrm{m}$ nanowires in Fig. 6(b). The dimensional information on length, width, and height of each nanowire was obtained from AFM sectional profiles [see the caption of Fig. 6(b)]. The information of resistance $R$ was found from the slope, as shown in Fig. 6(b). The coupled equations were solved to determine $\rho$ and $C$. We repeated these steps for all four possible pairs to find the average value for comparison with the literature value. The standard deviation was also calculated to find the uniformity of both $\rho$ and $C$. The $\rho$ and $C$ were averaged to be $(1.73 \pm 0.19) \times 10^{-7} \Omega \mathrm{m}$ and $(1.99 \pm 0.65) \times 10^{-13} \Omega \mathrm{m}^{2}$, respectively, for all four analyses. The electrical resistivity of the gold nanowire obtained is seven times higher than that of the bulk gold, $2.44 \times 10^{-8} \Omega \mathrm{m}$, found in literature. ${ }^{20}$ This 
result suggests that the confined geometry of the nanowires increases the electrical resistivity significantly. Using the Eq. (3) and the constant $C$, the contact resistances are calculated to be $152 \pm 50 \Omega$ and $205 \pm 67 \Omega$ for the $10 \mu \mathrm{m}$ and $5 \mu \mathrm{m}$ nanowires, respectively. The contact resistance $R_{C}$ takes $9 \%$ $(=152 \Omega / 1665 \Omega)$ and $15 \%(=205 \Omega / 1340 \Omega)$ of the total resistance for the $10 \mu \mathrm{m}$ and $5 \mu \mathrm{m}$ nanowires, respectively. In other words, the nanowire resistance $\mathrm{R}_{\mathrm{NW}}$ takes more than $85 \%$ in our experiment, which is dominant over the contact resistance $R_{C}$ in the total resistance $R$ of the nanowires.

The standard deviation is $11 \%\left[=\left(0.19 \times 10^{-7} \Omega \mathrm{m}\right)\right.$ $\left./\left(1.73 \times 10^{-7} \Omega \mathrm{m}\right)\right]$ for the electrical resistivity, while it is $\sim 30 \%(=50 \Omega / 152 \Omega)$ for the contact resistance. This higher variation in the contact resistance may be due to the irregular interfaces (and thus the cross section area) at the boundaries of gold pads in Fig. 6(a). Since $\Delta \mathrm{R}_{\mathrm{NW}} / \mathrm{R}_{\mathrm{NW}}=-\Delta \mathrm{w} / \mathrm{w}$ from Eq. (2) assuming that $\rho, l$, and $h$ are constant, the $\sim 10 \%$ variation in $\Delta R_{N W} / R_{N W}$ corresponds to the same variation in the width, or $\Delta \mathrm{w} / \mathrm{w}$. This result suggests that our two-layer indentation method is capable of creating uniform and reproducible nanowires up to $90 \%$, consistent with the AFM topographic result [see Figs. 5(a) and 5(b)]. The efficient removal of polymer residues by the dry etching process may contribute to the observed uniformity and reproducibility of the gold nanowires. The dry etching process also contributed to the clean contacts between the nanowire and electrodes.
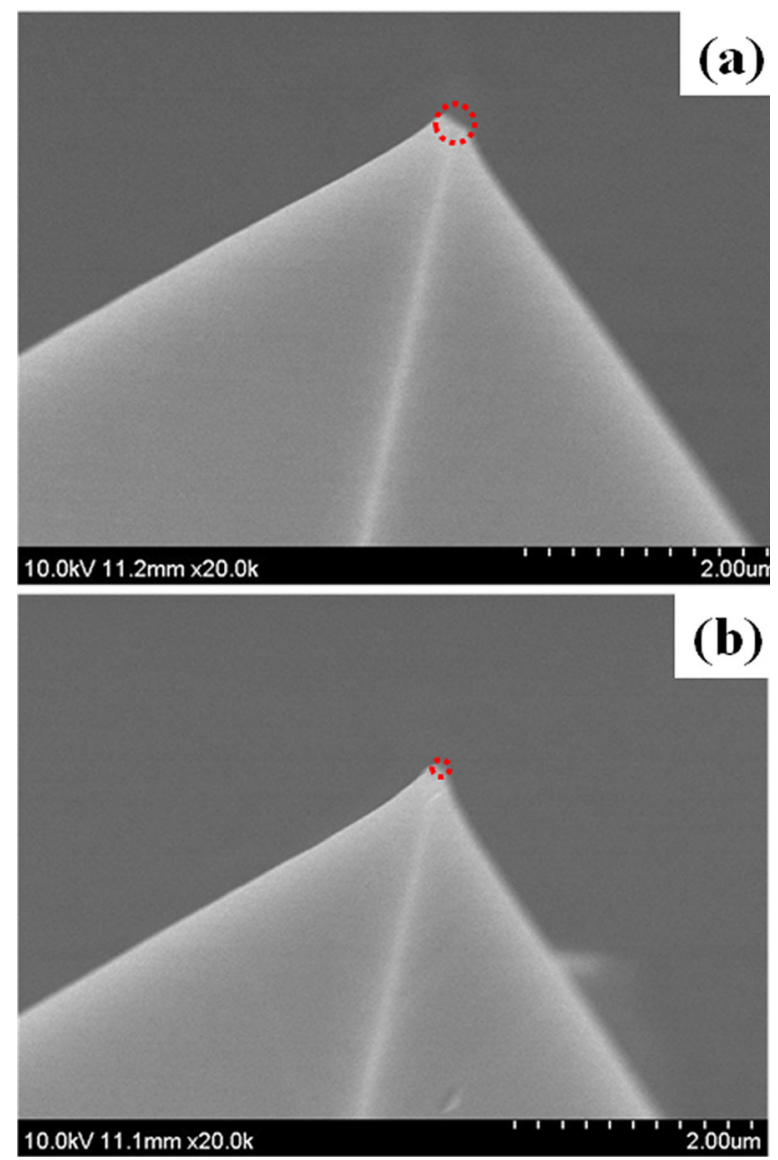

FIG. 7. (Color online) FE-SEM pictures of used AFM tip as (a) ploughing method and (b) the consecutive-hole-indentation method.
Another aspect worth mentioning is that the AFM feedback during the lateral tip movement between the successive indentations contributed to the result by enabling the indentation method to make indentation patterns with the same depth even with the height variations of the sample surface.

Finally, we investigated the damage to the AFM tip to compare the consecutive-hole-indentation method and the ploughing method when creating line grooves of the same depth. The tip damage was investigated after the fabrication of line grooves $15 \mu \mathrm{m}$ in length using each method, where each experiment was conducted with a brand new tip. The SEM measurements show that that the tip diameter used in the ploughing method [Fig. 7(a)] was approximately two times bigger than that used in the consecutive-hole-indentation method [Fig. 7(b)]. This result indicates that the consecutive-hole-indentation method caused less damage to the tip than the ploughing method.

\section{SUMMARY AND CONCLUSIONS}

Using a noncontact two-layer indentation method that we developed, we made uniform, two-dimensional reproducible nanostructures, solving one of the problems associated with AFM indentation. Through the topographic and electrical measurements of the fabricated gold nanowires, we demonstrated that uniform and reproducible nanostructures can be fabricated with conventional silicon tips without any special treatment such as diamond coating. This method minimized the tip damage by reducing the vertical and lateral forces acting on the tip when it presses on the substrate or moves between the thin gold layer-PMMA structures. Furthermore, the AFM indentation nanolithography method presented in this study is versatile. Although gold and PMMA were specifically used in this experiment, other combinations of metals and polymers can also be used.

\section{ACKNOWLEDGMENTS}

This research was supported by Basic Science Research Program through the National Research Foundation of Korea (NRF) funded by the Ministry of Education, Science and Technology (2010-0025043). This research was also partially supported by NSF DMR-1126854.

\footnotetext{
${ }^{1}$ J. Fujita, Y. Ohnishi, Y. Ochiai, and S. Matsui, Appl. Phys. Lett. 68, 1297 (1996).

${ }^{2}$ A Heuberger, J. Vac. Sci. Technol. B 6, 107 (1988).

${ }^{3}$ L. Bach, I. P. Reithmaier, A. Forchel, J. L. Gentner, and L. Goldstein, Appl. Phys. Lett. 79, 2324 (2001).

${ }^{4}$ P. M. Campbell, E. S. Snow, and P. J. McMarr, Appl. Phys. Lett. 66, 1388 (1995).

${ }^{5}$ P. M. Campbell and E. S. Snow, Semicond. Sci. Technol. 11, 1558 (1996). ${ }^{6}$ P. Avouris, T. Hertel, and R. Martel, Appl. Phys. Lett. 71, 285 (1997).

${ }^{7}$ G. Kwon, K. Ko, H. Lee, W. Lim, G.-Y. Yeom, S. Lee, and J. Ahn, J. Vac. Sci. Technol. B 29, 011034 (2011).

${ }^{8}$ H. J. Mamin and D. Rugar, Appl. Phys. Lett. 61, 1003 (1992).

${ }^{9}$ K. Wiesauer and G. Springholz, J. Appl. Phys. 88, 7289 (2000).

${ }^{10}$ A. A. O. Elkaseh, W. J. Perold, and V. V. Srinivasu, J. Appl. Phys. 108, 053914 (2010).

${ }^{11}$ Y. He, H. Dong, T. Li, C. Wang, W. Shao, Y. Zhang, L. Jiang, and W. Hu, Appl. Phys. Lett. 97, 133301 (2010).
} 
${ }^{12}$ C.-H. Shin, I.-S. Jeon, S.-H. Jeon, and Z. G. Khim, Appl. Phys. Lett. 94, 163107 (2009).

${ }^{13}$ B. Vasić, M. Kratzer, A. Matković, A. Nevosad, U. Ralević, D. Jovanović, C. Ganser, C. Teichert, and R. Gajić, Nanotechnology 24, 015303 (2013).

${ }^{14}$ K. Miyashita, S. Nishimura, T. Toyofuku, and J.-I. Shirakashi, J. Vac. Sci. Technol. B 27, 953 (2009).

${ }^{15}$ A. A. Tseng, J.-I. Shirakashi, S. Nishimura, K. Miyashita, and A. Notargiacomo, J. Appl. Phys. 106, 044314 (2009).
${ }^{16}$ J.-H. Hsu, C.-Y. Lin, and H.-N. Lin, J. Vac. Sci. Technol. B 22, 2768 (2004).

${ }^{17}$ Y.-J. Chen, J.-H. Hsu, and H.-N. Lin, Nanotechnology 16, 1112 (2005).

${ }^{18}$ C. Martín, G. Rius, X. Borrisé, and F. Pérez-Murano, Nanotechnology 16, 1016 (2005).

${ }^{19}$ S. Hu, A. Hamidi, S. Altmeyer, T. Köster, B. Spangenberg, and H. Kurz, J. Vac. Sci. Technol. B 16, 2822 (1998).

${ }^{20}$ J. D. Cutnell and K. W. Johnson, Physics, 6th ed. (Wiley, Hoboken, NJ, 2004), $582 \mathrm{pp}$. 